\title{
Subarachnoid hemorrhage presenting as TIA in the MCA territory
}

\author{
Ioan-Cristian Lupescu', Mirela Draghici', Ioana Gabriela Lupescư ${ }^{2,3}$, \\ Adriana Octaviana Dulamea ${ }^{1,3}$ \\ ${ }^{1}$ Neurology Department, Fundeni Clinical Institute, Bucharest, Romania \\ ${ }^{2}$ Radiology and Medical Imaging Department, Fundeni Clinical Institute, Bucharest, Romania \\ "Carol Davila" University of Medicine and Pharmacy, Bucharest, Romania
}

\begin{abstract}
A 71 yo woman treated with Lisinopril for HBP, was brought to our department for right-sided paresthesias, dysarthria and right-sided motor deficits. Onset of symptoms was sudden, about one hour prior to hospitalization. However, at the time of presentation, they were partially ameliorated. Clinical exam revealed right hemiparesis $4 / 5 \mathrm{MRC}$, right central facial palsy, BP of $150 / 80 \mathrm{mmHg}$ and $\mathrm{HR}$ of $78 / \mathrm{min}$ rhythmic. The patient presented similar transient episodes of right-sided paresthesias and dysarthria during the last month, but without addressing to a medical department. Medical exam and history pointed to a TIA in the left MCA territory. However, CT scan revealed a small intergyral left fronto-parietal subarachnoid hemorrhage. When asked, the patient denied any headache, nausea/vomiting, altered consciousness or recent history of head trauma, while clinical exam revealed no meningism. The patient was then transferred urgently to a Neurosurgery Department.
\end{abstract}

Keywords: subarachnoid hemorrhage, hemiparesis, TIA

Abbreviations (in alphabetical order):

$\mathrm{BP}$ - Blood pressure

CT - Computer tomography

HBP - High blood pressure

HR - Heart rate
MCA - Middle cerebral artery

MRC - Medical Research Council

SAH - Subarachnoid hemorrhage

TIA - Transient ischemic stroke

\section{INTRODUCTION}

Subarachnoid hemorrhage usually presents with a characteristic combination of symptoms, sudden severe headache being the most important and, in up to one third of cases, the only symptom (1). In one retrospective study of nontraumatic SAH cases during a 5-year period, most common presenting features were nausea/vomiting (77\%), headache (74\%), loss of consciousness (53\%) and nuchal rigidity (35\%) (2).

\section{CASE PRESENTATION}

A 71 yo woman treated with Lisinopril for HBP, was admitted to our department for right-sided par- esthesias, dysarthria and right-sided motor deficits. Onset of symptoms was sudden, about one hour prior to hospitalization. However, at the time of presentation, they were partially ameliorated.

Clinical exam revealed right hemiparesis $4 / 5$ MRC, right central facial palsy, BP of $150 / 80$ mmHg and HR of 78/min rhythmic.

The patient also reported similar transient episodes of right-sided paresthesias with dysarthria during the last month, without addressing to a medical department.

Medical exam and history pointed to a TIA in the left MCA territory. However, CT scan revealed a small intergyral left fronto-parietal subarachnoid hemorrhage. When asked, the patient denied any 

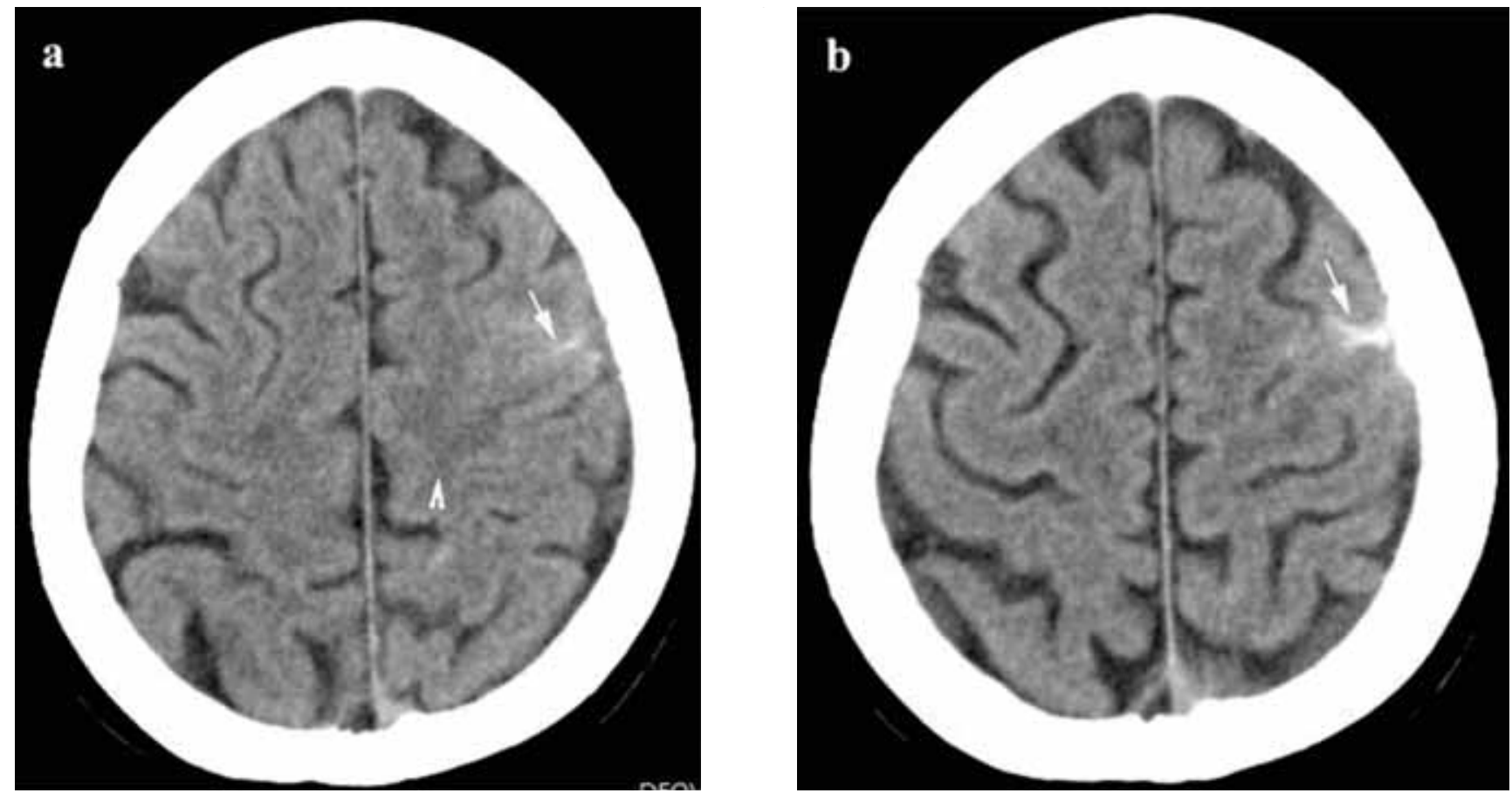

FIGURES 1 \& 2. Spontaneous hyperdense focal accumulation in the left frontal sulcus (arrow) with associated discrete hypodensity (edema - arrow head) in the frontal lobe white matter - CT appearance suggestive for limited subarachnoid hemorrhage.

headache, nausea/vomiting, altered consciousness or history of head trauma, while clinical exam revealed no meningism.

The patient was then transferred urgently to a Neurosurgery Department.

\section{DISCUSSIONS}

Our patient did not present any characteristic symptoms of $\mathrm{SAH}$, although clinical picture was in accordance with hemorrhage location. Moreover, she recalled similar transient episodes over the last month.

Such episodes could be regarded equivalents of the "sentinel headaches" or "warning leaks", which are thought to precede SAH onset. Some authors suggest abandoning the term "warning leaks", due to its confusing nature $(1,3)$. Based on a systematic review (Polmear), they appear to be a real entity, with varying incidence from near 0 to about $40 \%$ (4). However, their existence was not supported by a prospective study (Linn) of 148 patients with sudden severe headache (possible sentinel headache) and follow-up for 1 year (5). Another prospective study found no clinical and imaging difference between SAH cases with prior headache and those without (6).

Additionally, warning signs may be caused by the presence of expanding aneurysms. In one study, prodromal symptoms were found in $59 \%$ of patients with intracranial aneurysms, with an average of 6 days between last warning signs and hemorrhage (for MCA aneurysms) (7). In another study, warning signs were divided in 3 groups, based on possible etiology ( 1 - mass effect of expanding aneurysm, 2 - minor bleeds, 3 - spasm/occlusion), with shortest interval before hemorrhage onset occurring in group $2(\approx 10$ days), and thus requiring most urgent medical attention (8).

As for headache peaking within an hour, several clinical features were found to be predictive of SAH: age $\geq 40$ years, onset with exertion, arrival by ambulance, vomiting, witnessed loss of consciousness, nuchal rigidity/pain, $\mathrm{BP}>160 \mathrm{mmHg}$ (systolic) and/or $>100 \mathrm{mmHg}$ (diastolic). Presence of any of these features should prompt investigation for SAH (9).

It should be emphasized that SAH can be triggered during non-exertional activities, sometimes more frequently than during exertional activities (2). 


\section{REFERENCES}

1. Al-Shahi R., White P.M., Davenport R.J. et al. Subarachnoid haemorrhage. BMJ: British Medical Journal. 2006; 333(7561): 235-240.

2. Fontanarosa P.B. Recognition of subarachnoid hemorrhage. Ann Emerg Med. 1989;18(11):1199.

3. Davenport R. Acute headache in the emergency department. $J$ Neurol Neurosurg Psychiatry. 2002; 72 (Suppl 2): ii33-ii37.

4. Polmear A. Sentinel headaches in aneurysmal subarachnoid haemorrhage: what is the true incidence? A systematic review. Cephalalgia. 2003; 23(10): 935-941.

5. Linn F.H., Wijdicks E.F., van der Graaf Y. et al. Prospective study of sentinel headache in aneurysmal subarachnoid haemorrhage. Lancet. 1994; 344(8922): 590-593.
6. Linn F., Rinkel G., Algra A. et al. "The Notion of 'Warning Leaks' in Subarachnoid Haemorrhage: Are Such Patients in Fact Admitted with a Rebleed?" Journal of Neurology, Neurosurgery, and Psychiatry. 2000; 68(3): 332-336.

7. Waga S., Otsubo K., Handa H. Warning signs in intracranial aneurysms. Surg Neurol. 1975; 3(1): 15-20.

8. Okawara S.H. Warning signs prior to rupture of an intracranial aneurysm. J Neurosurg. 1973; 38(5): 575-580.

9. Perry J.J., Stiell I.G., Sivilotti M.L. et al. High risk clinical characteristics for subarachnoid haemorrhage in patients with acute headache: prospective cohort study. BMJ. 2010; 341: c5204

Conflict of interest: none declared Financial support: none declared 